\title{
Extraction of the Proton Parton Density Functions from the Combined HERA I Data using NLO QCD Fit
}

\author{
Voica A. Radescu ${ }^{1}$ on behalf of the $\mathrm{H} 1$ and ZEUS Collaborations \\ 1- Deutsches Elektronen Synchrotron (DESY) \\ Notkestrasse 85, Hamburg, D-22607 - Germany
}

\begin{abstract}
The precise combined HERA-I data set, of neutral and charged current inclusive crosssections for $e^{+} p$ and $e^{-} p$ scattering, is used as the sole input for a next-to-leading order (NLO) QCD parton distribution function (PDF) fit. The consistent treatment of systematic uncertainties in the combined data and the use of an improved theoretical model that takes into account the quark masses result in a new HERA PDF set. Model uncertainties, including those arising from parametrisation dependence, are also carefully considered. The resulting HERAPDF0.2 parton distributions have impressive precision compared to the global fits.
\end{abstract}

\section{Introduction}

Deep inelastic scattering (DIS) at HERA has been crucial to the exploration of proton structure and quark-gluon interaction dynamics. The two collider experiments, H1 and ZEUS, at HERA have recently ended the data taking process, after operating for 15 years. Running of HERA proceeded in two phases, HERA I, from 1992 to 2000, and HERA II, from 2003 to 2007. The preliminary results presented in this proceedings are based on data taken by both experiments during the HERA I period. HERA operated with an electron beam, $E_{e}=27.5 \mathrm{GeV}$ and a proton beam, $E_{p}$, of $820 \mathrm{GeV}$ until 1997 and of $920 \mathrm{GeV}$ afterwards, collecting about $120 \mathrm{pb}^{-1}$ of the $e^{ \pm} p$ scattering data per experiment, with the kinematic range extended to $0.045<Q^{2}<30000 \mathrm{GeV}^{2}$ and $6 \times 10^{-5}<x<0.65$. This yielded the most precise published double differential unpolarised neutral and charged current (NC and CC) DIS cross section measurements to date. The combination of these measurements resulted into a consistent and very precise data set with 110 systematic uncertainties from the separate H1 and ZEUS data sets and 3 sources of uncertainties from the combination procedure. The details of the combination procedure are described in [2].

In this proceedings a new set of parton distributions is determined, labeled HERAPDF0.2, using the combined data set as a sole input for an NLO QCD fit and an improved theoretical model which takes the quark masses into account. This study is part of a mid term strategy as it will be followed by combination and QCD analyses of even more accurate data from both HERA periods.

\section{QCD Analysis}

The QCD predictions for the structure functions are obtained by convoluting the PDFs with the calculable NLO coefficient functions. In the present analysis, the coefficient functions have been calculated using an improved theoretical scheme of general mass variable favour of Thorne and Roberts [3], as compared to the zero-mass variable flavour number scheme used for the previously released HERA PDFs, HERAPDF0.1 [4]. The PDFs are evolved using the DGLAP evolution equations at NLO in the $\overline{M S}$ scheme with the renormalisation 
and factorisation scales chosen to be the $Q^{2}$ scale $^{\text {a }}$. The strong coupling constant was fixed to $\alpha_{s}\left(M_{Z}\right)=0.1176$ [7], and variations in this value of \pm 0.002 have been considered. The following PDFs are parametrised: $x u_{v}, x d_{v}, x g$ and the $u$-type and $d$-type antiquarks $x \bar{U}$, $x \bar{D}$, where $x \bar{U}=x \bar{u}, x \bar{D}=x \bar{d}+x \bar{s}$. The input scale for the parametrisation is set to $Q_{0}^{2}=1.9 \mathrm{GeV}^{2}$ for the central fit, which is below the charm mass threshold, $Q_{0}<m_{c}$. The variation of the starting scale is considered as one of the model uncertainties, as well as the variations of the heavy quark masses which are set to the standard values of $m_{c}=1.4 \mathrm{GeV}$ and $m_{b}=4.75 \mathrm{GeV}$ for the central fit. The general functional form used for the PDF parametrisation is of the type

$$
x f(x)=A x^{B}(1-x)^{C}\left(1+D x+E x^{2}\right),
$$

with the normalisation parameters, $A_{u v}, A_{d v}, A_{g}$, constrained by the sum-rules. The $B$ parameters $B_{\bar{U}}$ and $B_{\bar{D}}$ are set equal, $B_{\bar{U}}=B_{\bar{D}}$, such that there is a single $B$ parameter for the sea distributions. Although heavy quarks are generated dynamically, the strange quark distribution is already present at the starting scale. It is assumed here that it can be expressed as $x$ independent fraction, $f_{s}$ of the $d$-type sea, $x \bar{s}=f_{s} x \bar{D}$ at $Q_{0}^{2}$. The further constraint $A_{\bar{U}}=A_{\bar{D}}\left(1-f_{s}\right)$, ensures that $x \bar{u} \rightarrow x \bar{d}$ as $x \rightarrow 0$. The strange fraction is chosen to be $f_{s}=0.31$ which is consistent with determinations of this fraction using neutrino induced di-muon production. The variation of it is considered as a model uncertainty. Only the parameters that significantly contribute to $\chi^{2}$ are left to vary. Additional requirements for the central fit are that all PDFs are positive definite and the valence quark approximation $d_{\text {valence }}>\bar{d}$ is satisfied at large $x$. The best fit results in a total of 10 free parameters, with $E_{u v} \neq 0$ and is chosen as the central fit.

The HERA measurements are at $W_{\min }^{2}=300 \mathrm{GeV}^{2}$ and $x_{\max }=0.65$, so that the target mass correction and higher twist contributions are believed to be small. In addition, only data above $Q^{2}=3.5 \mathrm{GeV}^{2}$ is used in the fit to remain in the region where perturbative QCD is valid. Variation of the latter is included as one of the model uncertainties. The model uncertainty error is estimated by adding in quadrature the difference between the central fit and each of the above mentioned model variations. This is added to the experimental uncertainty of the PDFs.

Besides the model uncertainties, an attempt is made here to quantify uncertainties related to the parameterisations of the PDF's. This dependence is assessed by considering all possible combinations for 11 parameter fits with $D$ and $E$ non-zero, which in fact do not represent a significant improvement in fit quality compared to the central fit. In addition, the criteria that all PDFs should be positive or that $d_{\text {valence }}>\bar{d}$ at high $x$ are no longer imposed for these fits. The uncertainty on the PDFs due to these parametrisation variations are estimated as an envelope which is formed as a maximal deviation at each $x$ value from the central fit.

The form of the $\chi^{2}$ is consistent with the one used for the combination of the data sets [2]. The consistency of the input data set and its small systematic uncertainties enable us to calculate the experimental uncertainties on the PDFs using the $\chi^{2}$ tolerance, $\Delta \chi^{2}=1$. In addition, the role of correlated systematic uncertainties is no longer crucial since these uncertainties are relatively small. Therefore, similar results are obtained when using either Offset or Hessian methods, or by combining all systematic uncertainties in quadrature. For

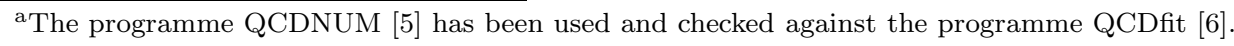



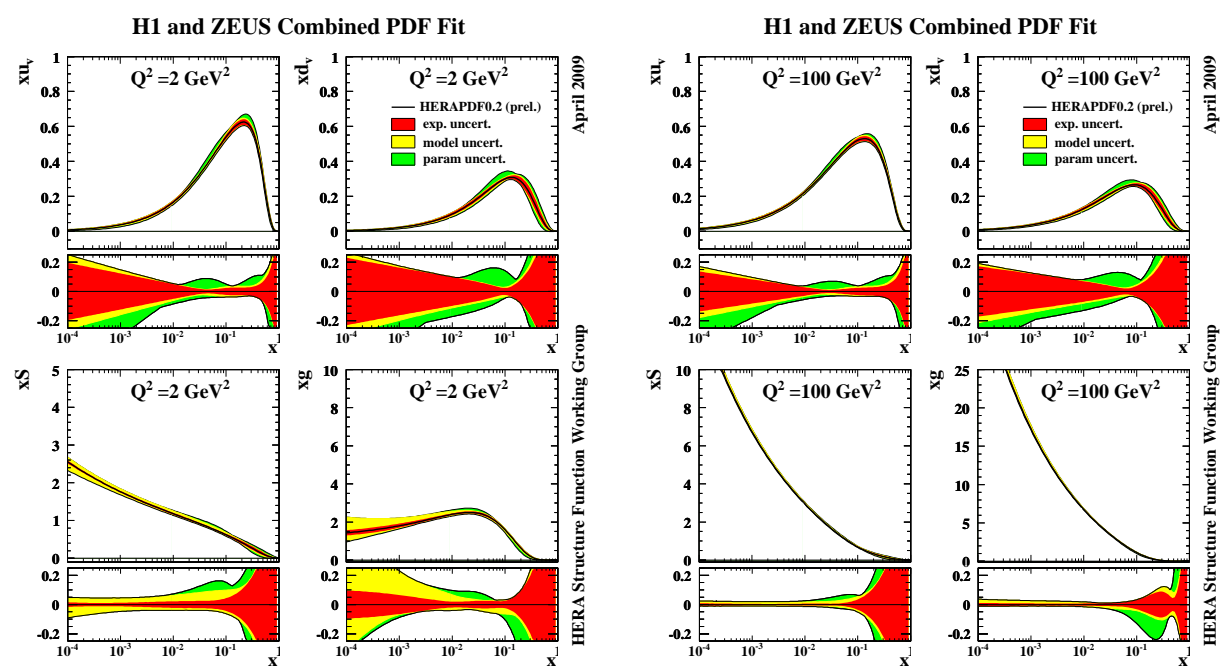

Figure 1: Figure shows the PDF distributions for the $x u_{v}, x d_{v}, x S$, and $x g$ with their relative uncertainties which include the experimental (red), model (yellow), and parametrisation (green) uncertainties at the $Q^{2}=2 \mathrm{GeV}^{2}$ (left) and $Q^{2}=100 \mathrm{GeV}^{2}$ (right).

HERAPDF0.2, the 110 systematic uncertainties are added in quadrature, and the 3 procedural sources of uncertainty are Offset, which is the most conservative choice to estimate the experimental uncertainty. The resulting $\chi^{2}$ per degree of freedom for the central fit is found to be $574 / 582$.

\section{$3 \quad$ Results}

The HERAPDF0.2 PDF sets are shown in Fig. 1 at $Q^{2}$ scales of 2 and $100 \mathrm{GeV}^{2}$, respectively. It can be observed that near the starting scale the shape of the gluon distribution is valence like and the errors of the sea and gluon distributions in the low $x$ region are dominated by the model uncertainties. The largest of which are due to the variations in the starting scale $Q_{0}^{2}$. The valence distributions are dominated by the parametrisation uncertainty and at high $x$ this uncertainty dominates all the distributions.

Figure 2 shows the summary plot for the HERAPDF0.2 displaying the gluon, sea and valence distributions at $Q^{2}=10 \mathrm{GeV}^{2}$. Figure 3 displays the comparison of the previous extracted HERAPDF0.1 to HERAPDF0.2. For a consistent comparison, only the experimental errors are shown. It can be observed that the resulting experimental uncertainty of the PDFs obtained from the new HERA I combined data set, is reduced compared to the ones of HERAPDF0.1. This is expected due to effect of the inclusion of the newly published HERA-I data of low and mid $Q^{2}$. In Fig. 4 the HERAPDF0.2 set is compared to the global fits of CTEQ6.6 [8] and MSTW08 [9] sets (at the 68\% confidence level) which also use a massive variable flavour number scheme. The precision of the HERAPDF0.2, which includes the experimental, model and parametrisation uncertainies, is impressive for the low- $x$ sea and gluon. However, further investigation to assess the PDF parametrisation uncertainty is needed by exploring various functional forms that could affect the low- $x$ region. 


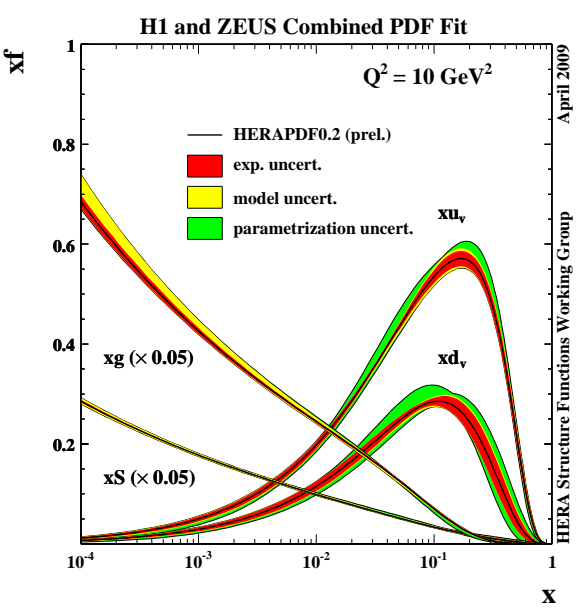

Figure 2: Figure shows the summary plot for the HERAPDF0.2 at the $Q^{2}=10 \mathrm{GeV}^{2}$. The shown PDFs are the gluon, sea (which are scaled by a factor of 0.05) and the valence distributions. The errors include the experimental (red), model (yellow) and the PDF parametrisation (green) uncertainties.

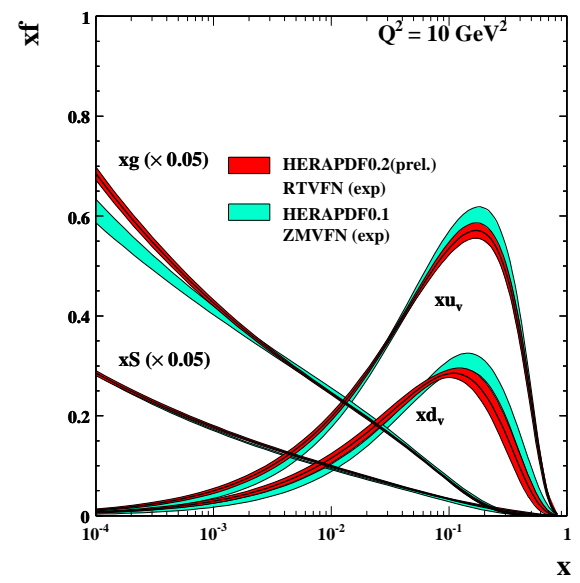

Figure 3: Figure shows a summary plot for direct comparison between the HERAPDF0.1 (cyan) and HERAPDF0.2 (red) at the $Q^{2}=10 \mathrm{GeV}^{2}$.

\section{Summary}

In this proceedings a new set of parton distributions is determined from an NLO QCD fit based on the precise combined HERA I data sets of neutral and charged current inclusive cross-sections for $e^{+} p$ and $e^{-} p$ scattering, and an improved theoretical model which takes the heavy charm and bottom masses into account. The consistent treatment of systematic uncertainties in the joint data set ensures that experimental uncertainties on the PDFs can 

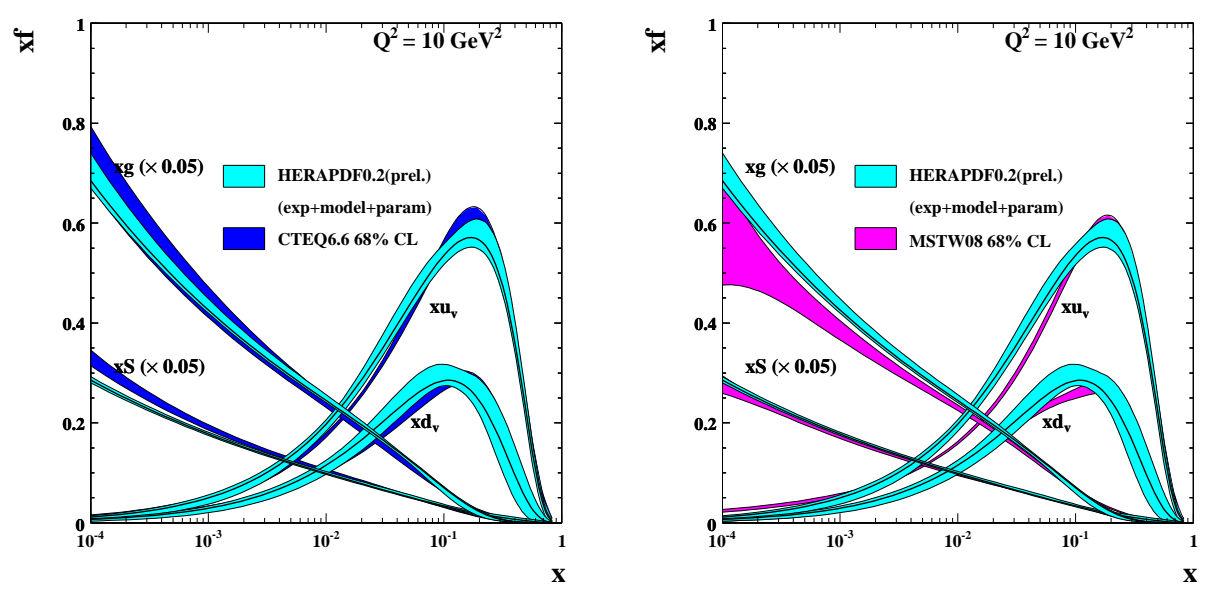

Figure 4: Figure shows the comparison of the HERAPDF0.2 (cyan) sets of PDFs to CTEQ6.6 (blue) and MSTW08 (magenta) at $Q^{2}=10 \mathrm{GeV}^{2}$. The CTEQ6.6 and MSTW08 PDF distributions are shown at $68 \%$ Confidence Level (CL). The HERAPDF0.2 error band includes the experimental, model and parametrisation uncertainties. The PDF sets shown are the gluon, sea, and valence distributions for up and down quarks.

be calculated without need for an increased $\chi^{2}$ tolerance. Model uncertainties, including those arising from parametrization dependence, have also been carefully considered and included in the total uncertainty estimate. The resulting HERA PDFs have impressive precision compared to the global fits.

\section{References}

[1] Slides:

http: //indico . cern. ch/contributionDisplay . py? contribId=86\&sessionId=22\&conf $I d=53294$

[2] E. Tassi, Proceedings of DIS 2009

[3] R. S. Thorne and R. G. Roberts, Phys. Lett. B 421 (1998) 303;

A. D. Martin et al. Eur. Phys. J. C 18 (2000) 117;

R. S. Thorne code, revised in 2008

[4] A. M. Cooper-Sarkar, Proceedings of DIS 2008, HERAPDF0.1 available at http://projects.hepforge.org/lhapdf/

[5] M. Botje, QCDNUM17.02, available at www.nikhef.nl/ h24/qcdnum

[6] C. Pascaud and F. Zomer, QCDfit, LAL preprint LAL 95-05; hep-ph/0104013

[7] Particle Data Group Listing (2008)

[8] P. M. Nadolsky, H.-L. Lai, Q.-H. Cao, J. Huston, J. Pumplin, D. Stump, W.-K. Tung, C.-P. Yuan; arXiv:0802.0007 [hep-ph]

[9] A. D. Martin, J. Stirling, R. S. Thorne, G. Watt; arXiv:0901.0002 [hep-ph] 